\title{
De novo mutations in PLXND1 and REV3L cause Möbius syndrome
}

\author{
Laura Tomas-Roca1,2, Anastasia Tsaalbi-Shtylik ${ }^{3}$, Jacob G. Jansen ${ }^{3}$, Manvendra K. Singh ${ }^{4,5}$, Jonathan A. Epstein ${ }^{4}$,

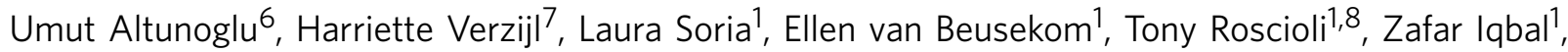 \\ Christian Gilissen ${ }^{1}$, Alexander Hoischen ${ }^{9}$, Arjan P.M. de Brouwer ${ }^{1}$, Corrie Erasmus ${ }^{7}$, Dirk Schubert ${ }^{10}$, \\ Han Brunner ${ }^{1,11}$, Antonio Pérez Aytés ${ }^{12}$, Faustino Marin², Pilar Aroca ${ }^{2}$, Hülya Kayserili6, Arturo Carta ${ }^{13}$, \\ Niels de Wind ${ }^{3}$, George W. Padberg ${ }^{7} \&$ Hans van Bokhoven ${ }^{1}$
}

Möbius syndrome (MBS) is a neurological disorder that is characterized by paralysis of the facial nerves and variable other congenital anomalies. The aetiology of this syndrome has been enigmatic since the initial descriptions by von Graefe in 1880 and by Möbius in 1888, and it has been debated for decades whether MBS has a genetic or a non-genetic aetiology. Here, we report de novo mutations affecting two genes, PLXND1 and REV3L in MBS patients. PLXND1 and REV3L represent totally unrelated pathways involved in hindbrain development: neural migration and DNA translesion synthesis, essential for the replication of endogenously damaged DNA, respectively. Interestingly, analysis of Plxnd1 and Rev3l mutant mice shows that disruption of these separate pathways converge at the facial branchiomotor nucleus, affecting either motoneuron migration or proliferation. The finding that PLXND1 and REV $3 L$ mutations are responsible for a proportion of MBS patients suggests that de novo mutations in other genes might account for other MBS patients.

\footnotetext{
${ }^{1}$ Department of Human Genetics, Radboud University Medical Center, Donders Institute for Brain, Cognition and Behaviour, PO Box 9101, Nijmegen 6500 HB, The Netherlands. ${ }^{2}$ Department of Human Anatomy and Psychobiology, School of Medicine, University of Murcia, 30100 Espinardo (Murcia), Spain. ${ }^{3}$ Department of Human Genetics, Leiden University Medical Center, P.O. Box 9600, 2300 RC Leiden, The Netherlands. ${ }^{4}$ Department of Cell and Developmental Biology, Cardiovascular Institute, Perelman School of Medicine at the University of Pennsylvania, 9-105 SCTR, 3400 Civic Center Boulevard, Philadelphia, Pennsylvania 19104, USA. ${ }^{5}$ Signature Research Program in Cardiovascular and Metabolic Disorders, Duke-NUS Graduate Medical School Singapore, National Heart Center Singapore, 8 College Road, Singapore 169857, Singapore. ${ }^{6}$ Medical Genetics Department, Istanbul Medical Faculty, Istanbul University, Millet Caddesi, Capa, Fatih 34093, Turkey. ${ }^{7}$ Department of Neurology, Radboud University Medical Center, Donders Institute for Brain, Cognition and Behaviour, PO Box 9101, Nijmegen 6500 HB, The Netherlands. ${ }^{8}$ The Kinghorn Centre for Clinical Genomics, Garvan Institute of Medical Research, Sydney, New South Wales 2010, Australia. ${ }^{9}$ Department of Human Genetics, Radboud University Medical Center, Radboud Institute for Molecular Life Sciences (RIMLS), PO Box 9101, Nijmegen 6500 HB, The Netherlands. ${ }^{10}$ Department of Cognitive Neuroscience, Radboud University Medical Center, Donders Institute for Brain, Cognition and Behaviour, PO Box 9101, Nijmegen 6500 HB, The Netherlands. ${ }^{11}$ Department of Clinical Genetics, Maastricht University Medical Center, PO Box 5800, Maastricht 6200AZ, The Netherlands. ${ }^{12}$ Dysmorphology and Reproductive Genetics Unit, Moebius Syndrome Foundation of Spain, University Hospital LA FE, Valencia 46540, Spain. ${ }^{13}$ Ophthalmology Unit, Department of Biomedical, Biotechnological and Translational Sciences (S.Bi.Bi.T.), University of Parma, via Gramsci 14, 43126, Parma, Italy. Correspondence and requests for materials should be addressed to N.d.W. (email: N.de_Wind@lumc.nl) or to G.W.P. (email: George.Padberg@radboudumc.nl) or to H.v.B. (email: Hans.vanbokhoven@radboudumc.nl).
} 
$\mathrm{M}$ öbius syndrome (MBS) (MIM 157900) is a rare congenital cranial dysinnervation disorder characterized by non-progressive facial palsy and impairment of ocular abduction, due to paralysis or weakness of the facial (n7) and abducens (n6) nerves, and frequently other cranial nerves ${ }^{1-6}$. Both intrauterine environmental factors and genetic causes have been proposed for the aetiology and pathogenesis of MBS. A disruption of blood vessel migration during development, which can be secondary to misoprostol ${ }^{7}$ or cocaine ${ }^{8}$ exposure during pregnancy, has been hypothesized to lead to hindbrain hypoxia, resulting in cranial nerve dysfunction ${ }^{9}$. A limited number of familial cases with an atypical manifestation of MBS have been reported $^{10-13}$. In addition, a founder mutation in HOXB1 has been associated with an autosomal recessive form of an MBS-like syndrome, with limited phenotypic overlap with typical MBS ${ }^{14}$. However, the vast majority of patients has a sporadic occurrence with a very low recurrence risk for siblings. This is consistent with either the involvement of environmental factors or with dominant de novo mutations as the underlying mechanism ${ }^{15}$.

The broad spectrum of neuropathological and neuroradiological findings suggests that MBS is due to a developmental defect of the entire rhombencephalon ${ }^{16,17}$, rather than an isolated cranial nerve disruption. This is in contrast to hereditary congenital facial palsy (HCFP), which is characterized by the isolated dysfunction of the facial nerve. Two HCFP loci have been identified: HCFP1 (MIM 601471) at chromosome 3q21-q22 (ref. 18) and HCFP2 (MIM 604185) at chromosome 10q21.3q22.1 (ref. 19). Extensive studies have been conducted to explain the HCFP phenotype and to pave the way for genetic studies in MBS, but analysis of genes in the HCFP1 and HCFP2 loci, including the prime candidate gene PLXND1 at chromosome 3q21-q22 (ref. 20), has so far not identified any causative mutations in HCFP and in MBS patients ${ }^{21}$.

In this study we undertake exome sequencing of two caseparent trios and six isolated patients with classical MBS to investigate the possibility of an underlying genetic cause. We identify de novo mutations in five different genes. For two of these genes, PLXND1 and REV $3 L$, additional de novo mutations in other MBS patients are identified. The causality of de novo PLXND1 and $R E V 3 L$ mutations for the neuropathological features of MBS is further supported by analysis of the respective knockout mice. Strikingly, for both heterozygous mutants we observe hypoplasia of the facial branchiomotor nucleus, which is consistent with the facial nerve weakness in MBS patients. Taken together, the present data establish de novo mutations as a cause for MBS, providing a rationale for exome sequencing in patient-parent trios to identify de novo mutations in other genes underlying MBS.

\section{Results}

Identification of de novo mutations by exome sequencing. We excluded all variants inherited from either parent in the two trios under the hypothesis of de novo mutations as the underlying cause of MBS (Supplementary Table 1). Two de novo variants were detected in patient 1 (P1) (Fig. 1a; reported previously ${ }^{17}$ ), one in the SPO11 gene (c.712T > C; p.Cys238Arg; NM_012444) and the other in the PLXND1 gene (c.5685C >A; p.Asn1895Lys; NM_015103) (Fig. 1a,b; Supplementary Fig. 1; Supplementary Tables 2 and 3). SPO11 is involved in meiotic recombination, and targeted disruption of this gene in mice results in fertility problems $^{22}$, but no features related to MBS. The occurrence of a de novo mutation in PLXND1 was more consistent with a role for this gene in MBS, given that PLXND1 was previously considered a strong candidate for HCFP1 and MBS ${ }^{20,21}$. Trio-exome sequencing in patient 2 did not reveal any de novo variant. A different analytical approach was taken for the six patients with isolated MBS and this was to Sanger sequence all candidate variants identified in the exome studies (Supplementary Tables 1 and 4). Sanger sequencing confirmed de novo variants, identified by exome sequencing, in IFT172, CCDC160 and REV3L, in three unrelated patients. The de novo missense mutation in the IFT172 gene (NM_015662) identified in patient 3 (Fig. 1; Supplementary Tables 2 and 3) is unlikely to be causative for the MBS phenotype, because biallelic mutations are associated with the unrelated Jeune and Mainzer-Saldino Syndromes in humans ${ }^{23}$. The de novo variant in the X-chromosomal CCDC160 gene of patient 4 (P4) (a male) is a c.501delA frameshift mutation in exon 2 (NM_001101357) (Supplementary Fig. 1; Supplementary Tables 2 and 3). Patient 5 (P5; Fig. 1c) carries a de novo variant in REV3L, c.1096+1G>A, affecting the canonical donor splice site in exon 10 (NM_002912) (Fig. 1d; Supplementary Fig. 1, Supplementary Tables 2 and 3). Aberrant splicing products were identified by quantitative reverse transcriptase-PCR analysis of REV3L messenger RNA obtained from Epstein-Barr virus-transformed lymphoblastoid cell lines (EBV-LCLs) of patient P5. Sequencing of these products revealed aberrant $R E V 3 L$ transcripts lacking either exon 9 or exons 9 and 10 , in addition to decreased levels of normal $R E V 3 L$ transcript (Supplementary Fig. 2).

Additional de novo mutations in the PLXND1 and REV3L. Next, we screened CCDC160, PLXND1 and REV3L by Sanger sequencing in a cohort of 103 MBS patients, including some HCFP patients who were not previously tested for PLXND1 (ref. 21). The screening identified four additional de novo mutations in unrelated patients, two affecting PLXND1 and two affecting REV3L (Fig. 1; Supplementary Fig. 1; Supplementary Tables 2 and 3). The PLXND1 mutations were identified in patients P9 (c.4454_4455GC>CA; p.Arg1485Pro) and P10 (c.3018C > T; p.Leu1006Leu). The mutation in P9 affects a conserved arginine residue in the GTPase-activating protein domain of the protein. The silent mutation in P10 is at a leucine residue in the IPT/TIG domain (immunoglobulin-like fold, plexins, transcription factors/transcription factor immunoglobin) that is characteristic for plexin proteins ${ }^{24}$. The two additional de novo mutations in REV3L were identified in patients $\mathrm{P} 11$ and $\mathrm{P} 12$. A de novo missense mutation c.1160A $>\mathrm{G}$ was identified in patient P11, predicting an amino-acid substitution p.Glu387Gly. In addition, patient P12 carried a nonsense mutation (c.2662A > T; p.Lys $\left.888^{\star}\right)$, which is predicted to result in a loss-of-function allele.

The absence of PLXND1, REV3L and CCDC160 truncating variants in NHLBI Exome Sequencing Project (ESP; http:// evs.gs.washington.edu/EVS/) and a low residual variant intolerance score for these genes of -1.30 (4.88th percentile), -2.1 (1.55th percentile) and 0.014 (54.98th percentile), respectively, are consistent with PLXND1, REV3L and CCDC160 being intolerant to loss-of-function mutations ${ }^{25}$. The amino acids that are affected by the missense variants in REV3L and PLXND1 are highly conserved throughout evolution (p.Glu387Gly (P11), p.Asn1895Lys (P1), p.Arg1485Pro (P9) and p.Leu1006Leu (P10)) (Supplementary Fig. 3).

The probability of identifying multiple de novo mutations in the same gene in a cohort of 103 individuals was calculated using the Poisson test and is 0.0001217 for REV3L $\left(P\right.$ value $\left.=2.4 \times 10^{-4}\right)$ and $9.34 \mathrm{e}-05$ for PLXND1 $\left(P\right.$ value $\left.=1.8 \times 10^{-4}\right)$. The presence of a mutation in the other allele in these patients was excluded by sequencing the coding exons of PLXND1 and REV3L. Screening of CCDC160 gene in our cohort of male MBS patients did not reveal additional de novo mutations.

PLXND1 deficiency affects neural fibres' structures. PLXND1 encodes a protein of 521 amino acids and is a member of the plexin family of proteins. Plexins bind to semaphorins (Semas), a 
a

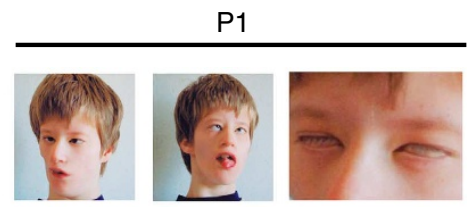

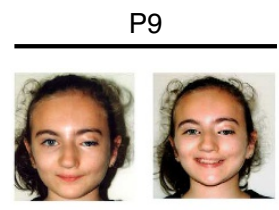

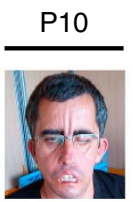

b

PLXND1 gene

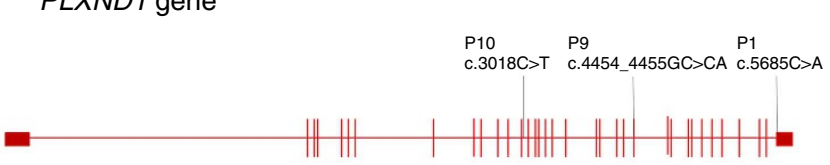

PLXND1 protein

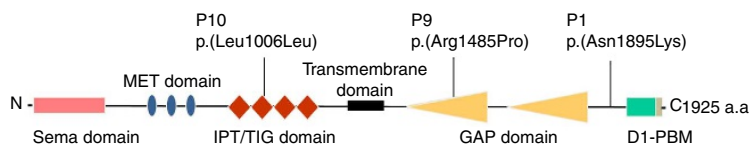

C

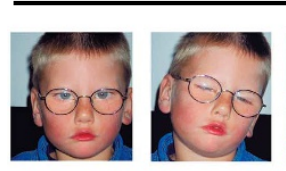

d

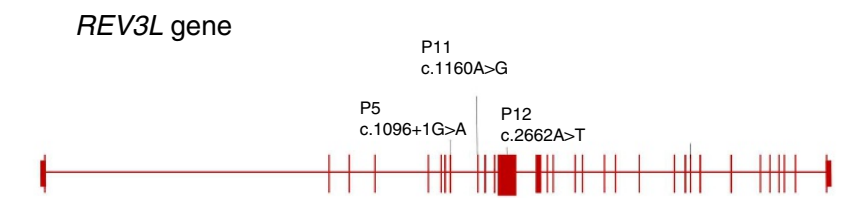

P5
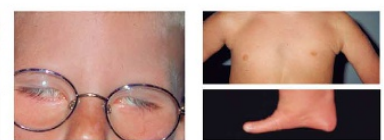

$\mathrm{P} 11$

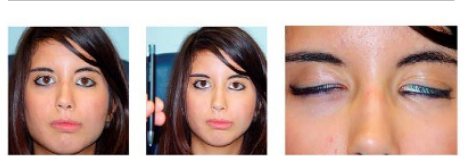

REV3L protein

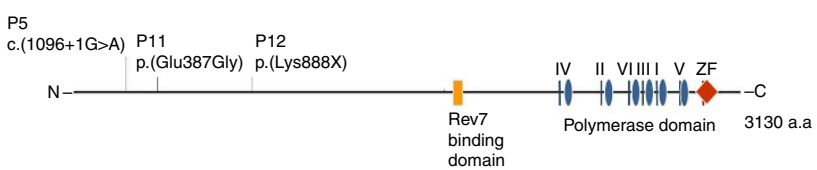

Figure 1 | Clinical features of Möbius syndrome patients and de novo mutations in PLXND1 and REV3L. (a) Clinical features of the patients carrying the mutations in PLXND1 (P1, P9 and P10). Patient P1 shows an impairment of ocular abduction in a relaxed facial position; she is not able to smile and she cannot close her eyes completely. Patient P9 in a relaxed facial position presents left upper facial paralysis. Patient P10 showing bilateral facial paralysis, inability to close his mouth and lagophthalmos. (b) Genomic structure of the PLXND1 gene and the PLXND1 protein structure and the position of de novo mutations. The different protein domains are depicted. GAP, GTPase-activating protein; IPT/TIG, immunoglobulin-like fold, plexins, transcription factors/transcription factor immunoglobin; D1-PBM, PDZ-binding motif; MET, mesenchymal-epithelial transition. (c) Clinical features of the patients carrying the mutations in REV3L (P5, P11 and P12). Patient P5 in a relaxed position, showing the characteristic inability of MBS patients to smile in the second photograph. Both eyes show incomplete closure. There is agenesis of the left pectoralis muscle (Poland variant) and absence of digits from the left hand. Patient P11 has both eyes fixed in straight position and a complete deficiency of both abduction and adduction. There is a complete inability to follow objects laterally. Furthermore, a bilateral facial nerve palsy is present producing a lagophthalmos on eye closure in both eyes, and oral rim asymmetry with an inability to smile. Patient P12 in a relaxed facial position. The ability to smile of P12 has been improved following plastic surgery at age of 13 years. Inappropriate closure of both eyes is still present. (d) Schematic structure of human REV3L gene (left) and REV3L protein (right). MBS-associated mutations detected in three patients (P5, P11 and P12) are indicated. The coloured bands represent the known domains of the protein. Rev7-binding domain, site of binding of the heterodimeric Rev3l partner Rev7; ZF, zinc finger.

large family of extracellular, secreted and membrane proteins ${ }^{26,27}$. PLXND1 is expressed in the vascular system ${ }^{20}$ and in specific structures of the central nervous system, such as the cranial and spinal ganglia, the cortical plate, the external granular layer of the cerebellum, the striatum and in dendritic cells ${ }^{28-30}$. Sema3E/PlexinD1 signalling is involved in the development of two descending forebrain tracts, the corticofugal and the striatonigral tracts ${ }^{31}$. To determine the effect of deficiency of PLXND1 in central nervous system development, we compared morphologic structures of the embryonic brain of wild-type (wt), heterozygous and homozygous PlexinD1 knockout mice at E16.5 (ref. 32). Histological comparison of the three genotypes revealed consistent hypoplasia of the corpus callosum, anterior commissure and fasciculus retroflexus in Plxnd1-I- mice (Fig. 2a). These three brain structures are bundles of neural fibres that connect brain areas. Both the corpus callosum and the anterior commissure allow interhemispheric communication, whereas the fasciculus retroflexus links the forebrain with the midbrain.

Plxnd1 mutant mice show a defect of neuronal migration. The facial nerve phenotype in Plxnd1 heterozygous and homozygous mice was investigated. Analogous to observations in brain autopsies of an MBS patient ${ }^{3}$, we observed a significant decrease in the number of motoneurons in the facial branchiomotor nucleus in homozygous mice as compared with wt mice (Unpaired $t$-test $P$ value $=0.0049$; Fig. 2b). The development of the cranial nerve undergoes a complex migration within the rodent hindbrain ${ }^{33}$ (Fig. 2c). Between E10.5 and E14.5, neurons in rhombomere 4 (r4) start migrating tangentially along the ventral midline, reaching $\mathrm{r} 5$ and $\mathrm{r} 6$. Then they migrate dorsally until the alar/basal plate boundary and begin to migrate radially to their final destination in the pial surface of $r 6$ (refs 33-35). To trace the nature of the decreased neuronal density of the FBM, we carried out an immunohistochemical analysis using the motoneuronspecific molecular marker Islet-1 in brains from E16.5 embryos (Fig. 2d). In line with this migration pattern, all FBM motoneurons reached $\mathrm{r} 6$ at E16.5 in wt mice (Fig. 2d). In sharp contrast, in all of the Plxnd $1^{+/}$and Plxnd $1^{-I-}$ mutant mice, a subpopulation of motoneurons was still located in $\mathrm{r} 4$ and $\mathrm{r} 5$ at E16.5. Collectively, these results support a causative role of a defect in motoneuron migration caused by a heterozygous Plxnd 1 mutation akin to the de novo PLXND1 mutations in MBS patients P1, P9 and P10. 
a

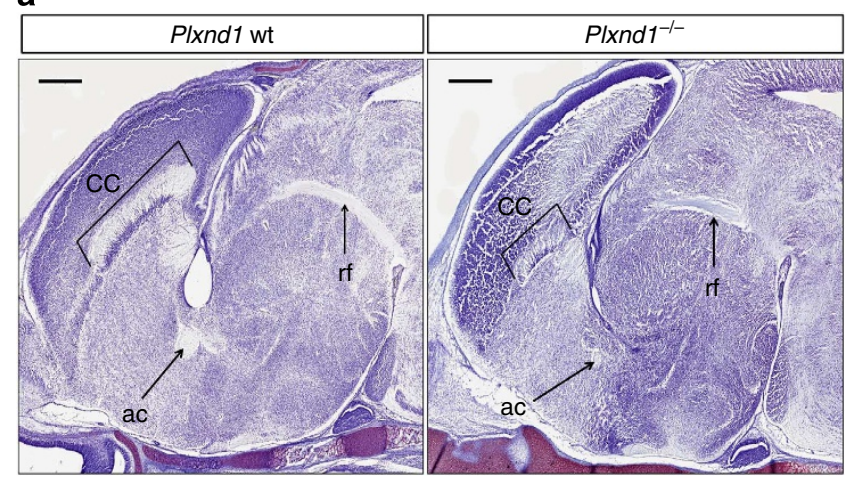

b

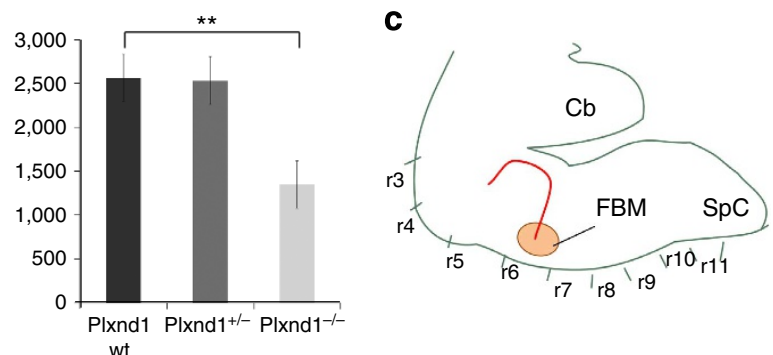

d
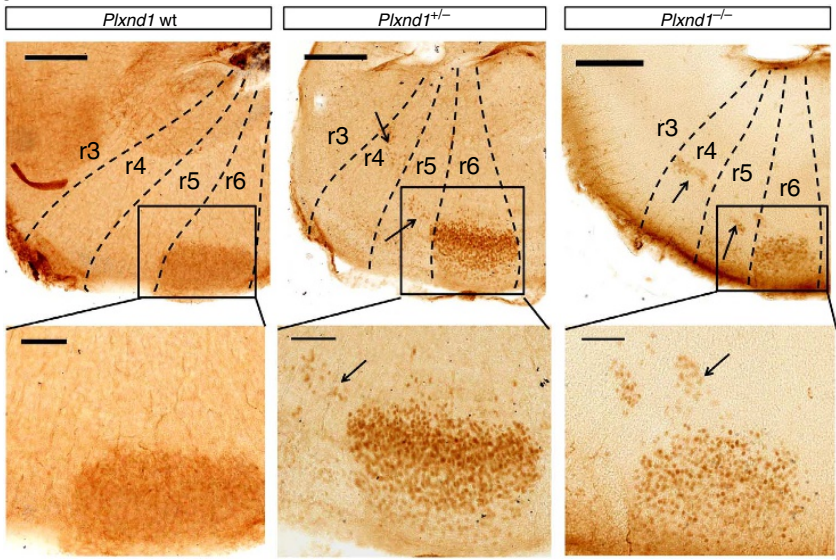

Figure 2 | Plxnd1 mouse brain characterization. (a) Paraffin sagittal sections of embryonic mouse brains processed with Nissl staining at E16.5. These two pictures are medial sections of a view of the corpus callosum (CC), the anterior commisure (ac) and the fasciculus retroflexus ( $r f)$. These three structures appear hypoplastic in Plxnd1 ${ }^{-/}-$mice. Scale bars,

$500 \mu \mathrm{m}$. (b) Graphic representation of the number of motoneurons in facial motor nucleus in wt, heterozygous and homozygous Plxnd1 knockout (mean \pm s.d.). Unpaired $t$-test was used to calculate the $P$ value $=0.0049$ $(N=5)$. (c) Schematic representation of the facial nerve migratory process along the hindbrain. The motoneuron migratory pathway is indicated with a red arrow. The rhombomeres ( $r 3-r 11)$ boundaries are marked with a line. $\mathrm{Cb}$, cerebellum; FBM, facial motor nucleus; SpC, spinal cord. The upper part in (d) shows the immunohistochemical staining of facial FBM motoneurons with anti-Islet- 1 antibody (dark brown). The rhombomere units ( $r 3-r 7)$ are marked with a dashed line. Black arrows point the motoneurons migration across the rhombomeres in the heterozygous and the homozygous Plxnd1 knockout mouse. The area of the facial motor nucleus is marked by the square. Scale bar, $300 \mu \mathrm{m}$. A detailed view of the facial motor nucleus and motoneurons migration is shown in the lower part of $\mathbf{d}$. Motoneurons appear outside the facial nucleus along the migratory path of the facial nerve in both the heterozygous and the homozygous genotypes. Scale bar, $100 \mu \mathrm{m}$. All reported $P$ values tested were calculated by the unpaired $t$-test using Graphpad software.
Rev3l heterozygous mice show hypoplasia of neural structures. The human REV3L protein acts as the catalytic subunit of DNA polymerase $\zeta$, a key protein in replication of endogenously or exogenously damaged $\mathrm{DNA}^{36,37}$. Consistent with the phenotypes of the Rev3l heterozygous mice, in situ hybridization data show that Rev3l is highly expressed in the developing embryonic brain around mid-gestation (Supplementary Fig. 4). Biallelic inactivation of Rev3l in mice leads to mid-term embryonic lethality. Rev3l-deficient mouse embryos display pleiotropic morphological abnormalities, but are invariably growth-retarded and display massive apoptosis, notably of the brain ${ }^{36}$. Analysis of the subarachnoid space at E16.5 in Rev3l $l^{+/-}$mice revealed a highly significant increase in subarachnoid volume ( $t$-test, $P$ value $=0.0047$; Fig. $3 \mathrm{a}$ ). In addition, Rev3l heterozygous mice at P0 showed significantly reduced hindbrain volumes as compared with wt ( $t$-test, $P$ value $=0.015$; Fig. $3 \mathrm{~b})$. We then analysed the facial motor nucleus at P0 (Fig. 3c,d). In contrast to the Plxnd 1 mutant mice, there was no evidence for a motoneuron migration defect in the Rev 3 l heterozygous embryos. Nevertheless, there was a strong decrease in the number of motoneurons in Rev3l heterozygous embryos ( $t$-test, $P$ value $<0.0001$; Fig. $3 \mathrm{~d}$ ). These data provide strong support for hypoplasia of the motor nucleus consequent to a heterozygous Rev3l mutation, similar as the REV3L mutation in MBS patients P5, P11 and P12.

Rev3l mutant MEFs displayed increase of DNA damage. The essential role of Rev3l in replication of damaged DNA has been extensively studied in vitro and in Rev3l-deficient cells and mice ${ }^{36-39}$. We wondered whether the neuronal defects in MBS patients and Rev3l heterozygous mice reflect partial replication defects at DNA carrying endogenous DNA lesions. To this aim, we analysed responses of immortalized Rev3l mutant mouse embryonic fibroblasts (MEFs) to Benzo[ $\alpha]$ pyrene diolexpoxide (BPDE), an agent that induces DNA damage that mimics bulky endogenous nucleotide lesions. Rev3l heterozygous MEFs displayed a slight, but significant, decrease in cell survival (Fig. 4a). To substantiate the effect of haploinsufficiency, we measured replication of BPDE-damaged DNA in these cells and found that Rev3l heterozygous cells displayed a slight but significant defect, suggesting the presence of replication stress (Fig. 4b, right panel). Since replication stress induces DNA damage-signalling cascades that initiate cellular senescence or apoptosis, we measured phosphorylation of the signalling proteins Rpa, Chk1 and H2AX (Fig. 4c) ${ }^{40}$. Remarkably, the Rev3l heterozygous cells displayed a level of DNA damage signalling that was almost as high as in Rev3l-deficient cells. These data suggest that, in Rev3l heterozygous mice, marginally under-replicated endogenous DNA lesions induce strong DNA responses, contributing to the observed neuronal phenotypes.

\section{Discussion}

We have identified de novo mutations in PLXND1 and REV3L in six unrelated sporadic MBS patients. The neuropathological alterations and other clinical findings in MBS patients correlate to those in the Plxnd1 and Rev3l mutant models. Thus, hypoplasia of the facial motor nucleus that was observed in both mouse models has also been documented in MBS patients ${ }^{3,16}$. In addition, several developmental anomalies that were previously reported in Plxnd1 knockout mice are mirrored in patients carrying a PLXND1 mutation, as well as other MBS patients. For example, craniofacial bone abnormalities observed in Plxnd1 knockout mice ${ }^{41,42}$ were also seen in patients $\mathrm{P} 1$ and $\mathrm{P} 9$, and which are commonly seen in other MBS patients ${ }^{17}$ (Supplementary Table 5). In addition, the vertebral defects in Plxnd1 mutant mice are reminiscent of the scoliosis in patient $\mathrm{P} 1$, and the 
a

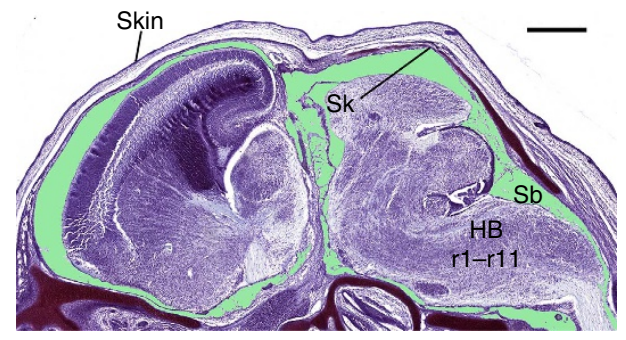

b

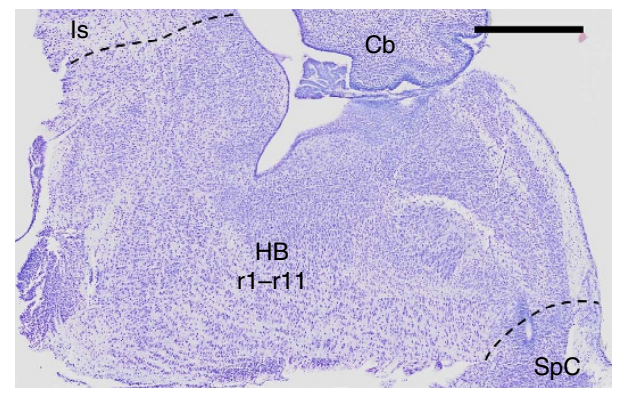

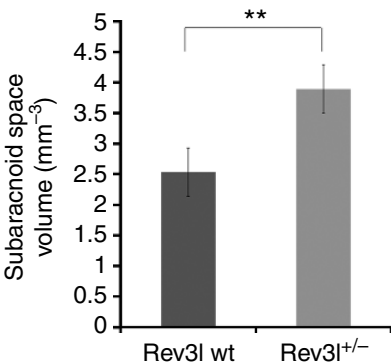

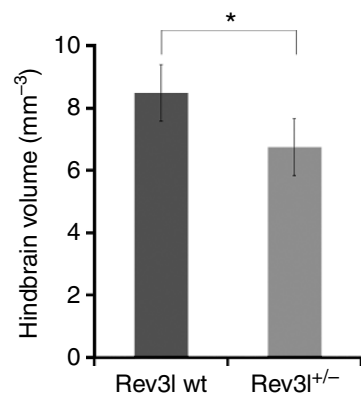

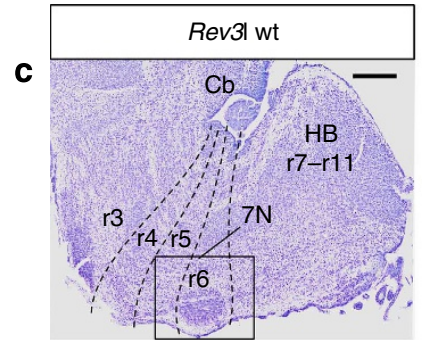

d

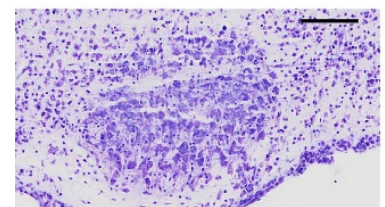

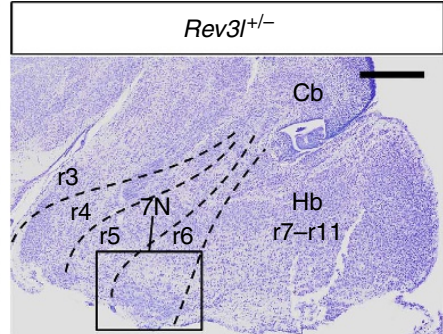

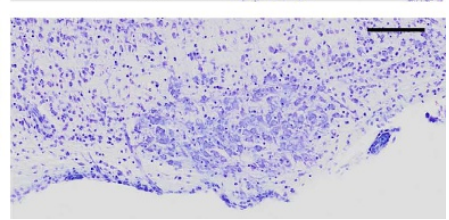

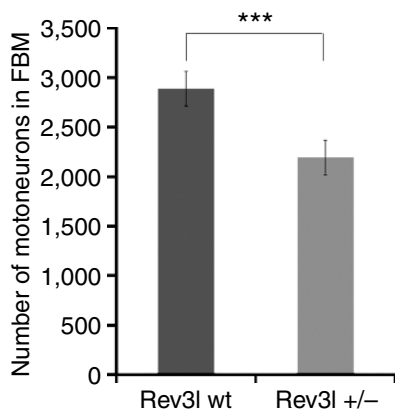

Figure 3 | Morphological analysis of the brains of Rev3l embryonic and newborn mice. (a) Example of a sagittal section of the embryonic mouse head at E16.5 stage used for subarachnoid volume rendering, processed with Nissl staining. The subarachnoid space is shown in green. Scale bar, $1 \mathrm{~mm}$. Right: subarachnoid volume rendering measures of both genotypes. In all Rev3/ heterozygous embryos the subarachnoid space is significantly increased as compared with wt embryos (Unpaired $t$-test $P$ value $=0.0047, N=5$ ). Scale bar, $500 \mu \mathrm{m}$ (b) Example of one of the hindbrain sagittal sections at P0 used for hindbrain volume rendering. We measured the volume of five wt and five heterozygous mice. The hindbrain boundaries are indicated by dashed lines. Scale bar, $500 \mu \mathrm{m}$. Right: Rev3l heterozygous mice show a significant decrease of hindbrain volume (Unpaired $t$-test $P$ value $=0.015, N=5$ ).

(c) Lateral hindbrain sections at the PO stage of a view of the facial motor nucleus, inside the square. Scale bar, $300 \mu \mathrm{m}$. (d) Higher magnification of the facial motor nucleus showing paucity of Rev3/ heterozygous motoneurons. Scale bar, $100 \mu \mathrm{m}$. Right: quantification of motoneurons in the facial motor nucleus, in each side of the hindbrain, in five wt and five heterozygous PO mice (mean \pm s.d.). The difference is statistically highly significant (Unpaired $t$-test $P$ value $<0.0001, N=10$ ). We do not show both genotypes because there are no visible differences between them. $5 \mathrm{~N}$, trigeminal nucleus; $7 \mathrm{~N}$, facial nucleus; $\mathrm{Cb}$, cerebellum; HB, hindbrain; Is, isthmus; r1-r11, rhombomeres $r 1$ to r11; Sb, subarachnoid space; Sk, skull; SpC, spinal cord. A P value smaller than 0.05 was considered to be statistically significant $\left({ }^{\star}\right)$. $P$ values smaller than $0.01\left(^{\star \star}\right)$ or $0.001\left(^{\star \star \star}\right)$ were considered highly significant.

haemorrhages seen in Plxnd1 mutant mouse resemble the facial haemangiomas seen in patient P1 (Supplementary Table 5). A possible causative de novo mutation was also identified in CCDC160. No PLXND1-coding mutations were identified in HCFP1 families, although their genetic defect maps to the PLXND1 locus ${ }^{20}$, suggesting the presence of regulatory mutations affecting the spatiotemporal expression of PLXND1 during embryonic brain development.

The clinical features of patients with heterozygous PLXND1 and $R E V 3 L$ mutations are highly variable with no obvious genotype-phenotype correlations. While the variable phenotype of patients $\mathrm{P} 1, \mathrm{P} 5$ and $\mathrm{P} 9-\mathrm{P} 11$ are consistent with the clinical criteria for $\mathrm{MBS}^{6,43}$, patient $\mathrm{P} 12$, who was identified as having a heterozygous nonsense mutation in $R E V 3 L$, only had bilateral facial paralysis and no weakness of the abducens nerves, suggestive of the restricted HCFP phenotype (Fig. 1; Supplementary Table 5). Thus, our data show that MBS and HCFP can be allelic conditions. In contrast to P12, the phenotype of patient P5, who was identified as having a heterozygous loss-of-function splice-site mutation in $R E V 3 L$, presented with a wide range of features in addition to the cranial nerve palsies, including absence of digits on the left hand, Poland anomaly, hearing loss and cardiac defect. This variability is highly reminiscent of the variable phenotype of completely Rev3l-deficient embryos. Rev3l deficiency may result in a stochastic ablation of cell lineages during embryonic 
a

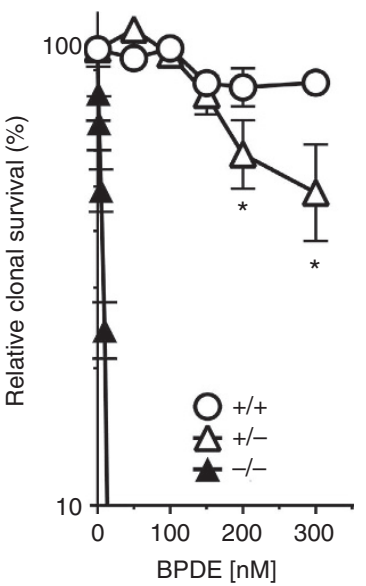

C

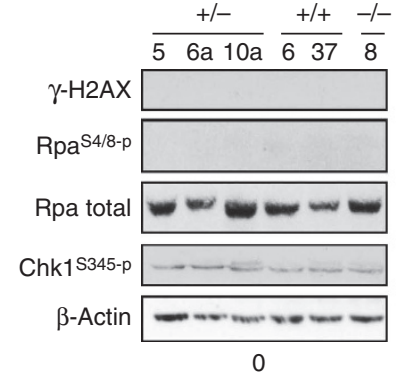

b
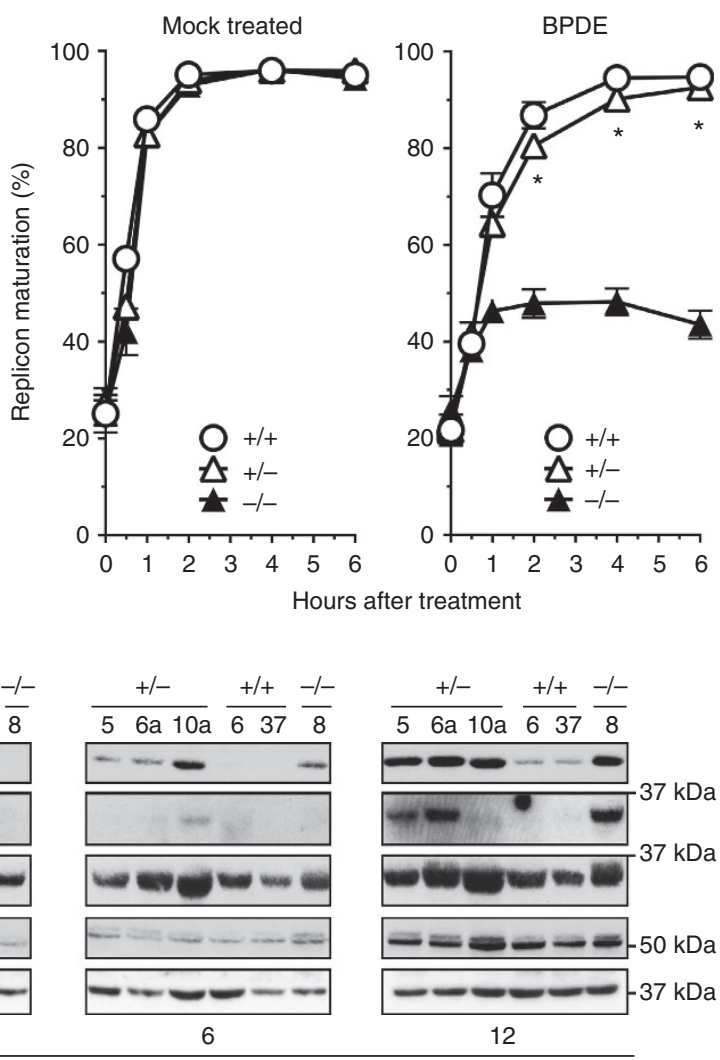

Hours after treatment

Figure 4 | Survival, replication of bulky DNA lesions and DNA damage signaling in Rev3l-mutated MEF lines. (a) Survival of wt $(+/+)$, Rev3l

heterozygous ( +/ - ) and Rev3l-deficient ( $-/-$ ) MEF lines after mock exposure, or after exposure to Benzo(a)pyrene diolepoxyde (BPDE). Rev3/ heterozygous MEFs are slightly sensitive to BPDE indicating haploinsufficiency. Experiments were performed in triplicate. Error bars, s.e.m. The asterisks indicate a significant difference between the wt and the heterozygous lines (unpaired $t$-test, $P<0.05$ ). (b) Representation of replication fork progression after exposure to BPDE, or mock exposure. Rev3l heterozygous MEFs display normal fork progression at undamaged DNA (mock treated, left panel), but a marginal defect in replication of bulky lesion-containing DNA (BPDE, right panel). Error bars, s.e.m. (c) Immunoblots to detect the phosphorylation of DNA damage-signalling markers upon treatment with BPDE. $\gamma$-H2AX: phosphorylated histone H2AX. Rpa ${ }^{S 4 / 8-P}: 32$ kDa subunit of single-stranded DNA-binding protein Rpa, phosphorylated at Ser 4 and/or Ser 8. Total levels of Rpa are somewhat variable consequent to slight differences in proliferation rate between the lines. Chk $1^{\mathrm{S} 354-\mathrm{P}}$ Checkpoint kinase-1, phosphorylated at Ser 354. $\beta$-actin: loading control. Rev3l-heterozygous MEFs display DNA damage signalling that is almost as strong as Rev3l-deficient MEFs.

development ${ }^{36}$. On the basis of these and our current data, it is conceivable that the spectrum of MBS symptoms reflects the stochastic loss of neuronal precursors, caused by replicative stress at endogenous DNA lesions and consequent DNA damage responses, during embryonic development. Likewise, the variable clinical presentation that is seen for PLXND1 mutations might be due to similar stochastic disruptions of motoneuron migration to the FBM nucleus.

Exome sequencing has detected causative de novo mutations in two (or three including the CCDC160 mutation) in the initial cohort of eight MBS patients. This result is of general interest, as several studies have suggested previously that vascular disruptions during the first trimester of pregnancy rather than genetic mechanisms are the leading cause of $\mathrm{MBS}^{5-7,44}$. While vascular disruptions caused by various teratogens can explain the MBS phenotype in some patients, our current data indicate that exome sequencing in parent-patient trios is a powerful approach for identifying causative mutations in MBS. It is to be expected that whole-genome sequencing will deliver an even higher yield when focusing on de novo mutations in coding and noncoding regions ${ }^{45}$. That only three de novo mutations were identified in PLXND1 and three in REV3L in our cohort suggests a low frequency of mutations in these genes as a cause of MBS, implying genetic heterogeneity and intrauterine insults as causative events in other MBS patients. We expect that the implementation of whole-exome sequencing and whole-genome sequencing of parent-patient trios in a diagnostic setting will further dissect the aetiology of MBS.

\section{Methods}

Patients. We identified eight isolated Möbius patients in this study. They were the only affected individuals in their families. These patients (patients 1-8) were selected from a cohort of patients with the pathognomonic signs of $\mathrm{MBS}^{6,43}$ : facial and abducens palsy ${ }^{17}$. Clinical details of these patients and other patients carrying de novo mutations (P9, P10, P11 and P12) are given in the Supplementary Table 5 and in Fig. 1a. Informed consent was obtained from all the subjects involved in this study, and all procedures were in accordance with the ethics of the World Medical Association Declaration of Helsinki. Ethical approval for the reported studies was obtained from the Medical Ethical Committee Arnhem-Nijmegen, from the University of Parma, the University of Istanbul and University Hospital LA FE, Valencia.

Whole-exome sequencing. Exome sequencing was performed on DNA from peripheral blood, using the SureSelect Human All Exon v2 50 Mb Kit (Agilent, Santa Clara, CA) followed by multiplexed analysis on a SOLiD 4 System sequencing slide (Life Technologies, Carlsbad, CA) as described previously ${ }^{46}$. Colour space reads were mapped to the hg19 reference genome with the SOLiD BioScope version 1.3 software, which utilizes an iterative mapping approach. Sequence analyses from all the patients are summarized in Supplementary Table 4. Statistical single-nucleotide variants were subsequently called by the Di-Bayes algorithm with high call stringency. Called variants were combined and annotated with a custom 
analysis pipeline. The total number of variants identified in each patient are summarized in Supplementary Table 1. Of these variants, some were located in the exonic sequences or located in canonical splice sites, defined as the dinucleotides up- and downstream of exonic sequence (Supplementary Table 1).

Under the hypothesis of de novo mutations as the underlying cause of MBS syndrome, we excluded all variants inherited from either parent in the two trios (Supplementary Table 1). From the remaining variants in these two patients, we excluded all synonymous variants, as well as variants present in (single-nucleotide polymorphism database) dbSNP (v138) or in our in-house-sequenced exome database (5,031 exomes), and variants in noncoding regions with the exception of canonical splice-site sequences. Manual inspection of mapped reads excluded additional, likely false positive, variants. After Sanger sequencing, only two variants were validated de novo mutations in one of the trios.

The approach for the six isolated cases was slightly different. For these, we applied a filtering scheme excluding variants observed in dbSNP (v138) and in our in-house database $(5,031$ exomes (Supplementary Table 1). We further analysed all truncating variants, missense variants with a PhyloP score $>3$ or those affecting genes with a possible involvement in MBS, as suggested by the gene ontology term or mouse mutant phenotype. Candidate variants were validated by Sanger sequencing, and parental DNAs were tested for de novo occurrence of the respective variant (Supplementary Tables 2 and 3).

The reference gene sequences referred to in this study are available in the RefSeq database under the following accession code: PLXND1, NM_015103; REV3L, NM_002912; CCDC160, NM_001101357; SPO11, NM_012444 and IFT172, NM_015662.

Mutation analysis. Primer sequences were designed using Primer3 software (http://frodo.wi.mit.edu/) encompassing the candidate variants. To exclude additional changes in either REV3L, PLXND1 or CCDC160, we designed primers for the amplification of all exons and intron-exon boundaries of REV3L (Genbank ID NM_002912), PLXND1 (Genbank ID NM_015103) and CCDC160 (Genbank ID NM_NM_001101357). Sequences of primers are shown in Supplementary Table 6. PCR was performed on $50 \mathrm{ng}$ of genomic DNA with Taq DNA polymerase (Invitrogen, Carlsbad, CA). A nucleofast 96 PCR plate (Clontech Lab, Mountain View, CA) was used to purify the PCR amplicons, according to the manufacturer's protocol. Sequence analysis was performed with the ABI PRISM Big Dye Terminator Cycle Sequencing V3.1 ready Reaction Kit and the ABI PRISM 3730 DNA Analyzer (Applera Corp, Foster City, CA). PCR products were sequenced by Sanger sequencing and analysed using Vector NTI software (Life Technologies).

Cell culture. EBV-LCL cells from patient P5 and five controls were cultured to a density of $0.5 \times 10^{6}$ cells per ml. After incubation for $4 \mathrm{~h}$ at $37^{\circ} \mathrm{C}$, cells were harvested by centrifugation at $200 \mathrm{~g}$ for $5 \mathrm{~min}$ at room temperature, washed once with PBS, pelleted by centrifugation at $200 \mathrm{~g}$ for $5 \mathrm{~min}$ at room temperature and snap frozen in liquid nitrogen. MEFs were obtained from E13.5-day-old embryos of intercrossed Rev3 ${ }^{+/-}, p 53^{+/-}$mice. The embryos were finely minced, trypsinized and the resulting MEFs were cultured in DMEM supplemented with $10 \%$ fetal calf serum and antibiotics until spontaneous immortalization ${ }^{47}$.

First-strand synthesis. EBV-LCL cells were harvested by centrifugation at $6,000 \mathrm{~g}$ at room temperature. RNA isolation was performed using the NucleoSpin RNA kit (Qiagen, Hilden, Germany) according to the manufacturer's protocols. RNA was reverse transcribed using the iScript cDNA synthesis kit (Bio-Rad Laboratories, Hercules, CA, USA) according to the manufacturer's protocol. Complementary DNA (cDNA) was purified with NucleoSpin extract II (Qiagen) according to the manufacturer's protocol.

Quantitative PCR (QPCR) analysis. SYBR green-based real-time QPCR expression analysis was performed on an Applied Biosystem Fast 7,500 System machine. Primer sequences around exon 10 were designed using Primer3 software (http://frodo.wi.mit.edu/) (Supplementary Table 6). Primers encompassed at least one boundary between two exons. GUSB was used as a reference gene ${ }^{48}$. QPCR quantifications were performed in duplicate on the equivalent of $5 \mu \mathrm{g}$ of total RNA from the first-strand synthesis and included a water control. Experimental threshold cycles $(\mathrm{Ct})$ values were within the range of cDNA dilutions used to validate the primers. The melt curves of all PCR products showed a single PCR product. All water controls were negative. Differences in expression of the REV3L alleles were calculated by the comparative Ct or $2^{\Delta \Delta \mathrm{Ct}}$ method ${ }^{49}$. The $P$ value was derived from the standard score ( $Z$-value) as compared with the normal distribution of the five controls. We used an $\alpha$-level of 0.05 , because only one gene was assessed.

Reverse transcriptase-PCR. cDNA from patient 1 EBV-LCLs and one control were used to amplify REV3L. The first pair of primers was designed surrounding the canonical splice-site mutation (c.1096 + 1G > A) in exon 10, and a control pair of primers was designed within exon 14 (see Supplementary Table 6).
Mouse breeding. Plxnd1 heterozygous mice were maintained on a C57BL/6 background and mated to generate Plxnd1 knockout and heterozygous embryos (male and female) for analysis. Plxnd1 null and wt mouse embryos (E16.5) were harvested from timed matings. Noon was designated E0.5. Embryos were genotyped as described ${ }^{32}$. All Plxnd1 mouse work was performed according to the University of Pennsylvania animal care guidelines.

C57BL/6 wt, Rev $3 l$ heterozygous and Rev3l-deficient mice and embryos were described before ${ }^{36}$. C57Bl/6 Rev3l heterozygous mice were mated to generate male and female embryos and newborn mice for analysis. Rev $3 l$ heterozygous and wt mouse embryos (E16.5) were derived from timed matings. Noon was designated E0.5. Embryos were genotyped as described ${ }^{36}$. Mice were treated using Federation of Laboratory Animal Science Associations (FELASA) standards and mouse experiments were performed under permit DEC 11108 from the animal experiment committee of the Leiden University Medical Center.

Mouse tissue preparation. Seven Plxnd1 wt, seven Plxnd1 heterozygous and seven Plxnd1 homozygous embryos at E16.5 (stage corroborated according to Theiler ${ }^{50}$ ) were killed and brains were dissected in PBS at room temperature and fixed in $4 \%$ paraformaldehyde at $4{ }^{\circ} \mathrm{C}$ overnight. The next day, brains were washed in PBS, dehydrated in ethanol and stored at $-20^{\circ} \mathrm{C}$. Brains were processed for paraffin and agarose embedding and sectioned into 16- and 90- $\mu \mathrm{m}$ slices using a microtome and vibratome, respectively.

We analysed two different stages to compare phenotypic aberrations. Five wt and five Rev3l heterozygous embryos at E16.5 (stage corroborated according to Theiler ${ }^{50}$ ) and five wt and five Rev $3 l$ heterozygous P0 newborn pups were killed and fixed by immersion in phosphate-buffered $4 \%$ paraformaldehyde $(0.1 \mathrm{M}$; $\mathrm{pH} 7.4)$ at $4{ }^{\circ} \mathrm{C}$ for $24 \mathrm{~h}$. Fixed brains and heads were embedded in paraffin and they were sectioned into $16-\mu \mathrm{m}$ slices using a microtome.

Tissue staining. Seven Plxnd1 wt, seven Plxnd1 heterozygous and seven Plxnd1 homozygous embryos at E16.5, five Rev3l wt and five Rev3l heterozygous embryos at E16.5 (stage corroborated according to Theiler ${ }^{50}$ ) and five Rev3l wt and five Rev3l heterozygous $\mathrm{P} 0$ newborn pups were embedded in paraffin, sectioned and were stained with Nissl stain according to routine protocols ${ }^{51}$.

Immunohistochemistry. Two Plxnd1 wt, two Plxnd1 heterozygous and two Plxnd1 homozygous embryos were embedded in agarose and processed for immune histochemistry with the Islet-1 antibody (catalogue number $39.4 \mathrm{D} 5$ ) obtained from the Developmental Studies Hybridoma Bank (University of Iowa, Iowa City). Sections were washed in PBS and then treated with $0.1 \%$ hydrogen peroxide in PBS for $1 \mathrm{~h}$ in the dark to inactivate endogenous peroxidase activity. After several rinses in PBT (PBS with $0.2 \%$ Triton X-100), sections were blocked with $0.5 \%$ goat serum, $0.2 \%$ BSA and $0.2 \%$ Triton X-100 (Sigma, St Louis, MO, USA) in PBS for $4 \mathrm{~h}$ and then incubated overnight at $4{ }^{\circ} \mathrm{C}$ with monoclonal antibody anti-Islet-1 (1:50 in blocking solution). Subsequently, sections were incubated with biotinylated goat anti-mouse secondary antibody (1:300, $2 \mathrm{~h}$ incubation; BA-9200, Vector Laboratories, Burlingame, CA, USA), and then with streptavidin/horseradish peroxidase complex (1:200, $2 \mathrm{~h}$ incubation; PK-4000, Vectastain-ABC kit; Vector Laboratories). All antibodies were diluted in the same blocking solution as the primary antibody. The histochemical detection of the peroxidase activity was carried out using $0.03 \%$ diaminobenzidine and $0.005 \%$ $\mathrm{H}_{2} \mathrm{O}_{2}$. After immunoreaction, the sections were mounted, dehydrated and then coverslipped with Eukitt (Fluka, Buchs, Switzerland).

Quantification of motoneurons. We counted the total number of motoneurons presents in the facial motor nucleus in paraffin sagittal sections processed with Nissl staining. We used five Plxnd1 wt, five Plxnd1 heterozygous and five Plxnd1 homozygous mice at E16.5 and five Rev3l wt and five Rev3l heterozygous P0 newborns, and we counted separately the number of motoneurons from the right and left hindbrain sides (around 12 sections each side) (20-25 slices total per animal). For the counting we used the open-source ImageJ Cell Counter software ${ }^{52}$. Differences in the number of motoneurons between the heterozygous, homozygous and the wt mice were calculated by a comparative $t$-test, using Graphpad Software (http://graphpad.com/quickcalcs/ttest1.cfm).

Brain structures volume rendering. We performed volume rendering of image stacks on serial light microscopic sections of $16 \mu \mathrm{m}$ using the open-source ImageJ TrakEM2 (ref. 53). For the volume rendering of the subarachnoid space around 100 sagittal slices of each of the five Rev3l wt and five Rev $3 l$ heterozygous mice at E16.5 were used. This embryonic stage was selected because the calcification of the cranial bones is not completed yet allowing the sectioning. For hindbrain volumes comparison, around 80 slices of each of the five Rev3l wt and five Rev3l

heterozygous brains at $\mathrm{P} 0$ were used. After alignment of all images from each brain respectively, we highlighted the areas of interest in each section to measure their volumes. The boundaries of the hindbrain were delineated according to the Allen Developing Mouse Brain Atlas from rhombomere r1 until r11, including the cerebellum. 
Survival assay. $\operatorname{Rev} 3 l$ survival assays were conducted with $\operatorname{Rev} 3 l^{+/+}, \operatorname{Rev} 3 \mathrm{l}^{+/-}$ and $\operatorname{Rev} 3 l^{-1-}$ MEFs. In each well of a six-well plate, $0.5 \times 10^{6}$ exponentially growing cells were seeded in MEF medium (DMEM supplemented with $10 \%$ fetal calf serum, glutamax and antibiotics) and cultured overnight. Then, the cells were exposed to $0-300 \mathrm{nM}$ BPDE (Biochemical Institute for Environmental Carcinogens, Grosshansdorf, Germany) that was dissolved in anhydrous tetrahydrofuran (THF, Sigma-Aldrich) for $60 \mathrm{~min}$ in serum-free medium at $37^{\circ} \mathrm{C}$. The cells were trypsinized and seeded in MEF medium at clonal densities in 9-cm dishes. After culturing for $12-14$ days at $37^{\circ} \mathrm{C}$, cell clones were fixed, stained with methylene blue and the number of cell clones was counted $(n=3)$. The number of clones after mock treatment was set at $100 \%$. The data were obtained with two and three independent $\operatorname{Rev} 3^{+1+}$ and $\operatorname{Rev} 3^{+1-}$ MEF lines, respectively, and one $\operatorname{Rev} 3^{-1-}$ MEF line.

Replication progression assay. Progression of replicons using the Alkaline DNA unwinding assay was determined following seeding of $5 \times 10^{4}$ exponentially growing cells per well in a 24-well plate and culturing overnight in MEF medium at $37^{\circ} \mathrm{C}$. The cells were mock treated with the solvent THF or exposed to $500 \mathrm{nM}$ $\mathrm{BPDE}$ in serum-free medium for $15 \mathrm{~min}$ at $37^{\circ} \mathrm{C}$. The medium was removed, cells were washed once with $150 \mathrm{mM} \mathrm{NaCl}$ and pulse labelled with $\left[{ }^{3} \mathrm{H}\right]$ thymidine $\left(2 \mu \mathrm{Ci} \mathrm{ml}^{-1} ; 76 \mathrm{Ci} \mathrm{mmol}^{-1}\right)$ in MEF medium for $15 \mathrm{~min}$ at $37^{\circ} \mathrm{C}$. MEF medium with radioactive thymidine was removed and the cells were cultured in MEF medium supplemented with $10 \mu \mathrm{M}$ cold thymidine. At different times after pulse labelling, the local unwinding of DNA ends of elongating replication forks, the separation of single-stranded DNA and double-stranded DNA (dsDNA) using hydroxylapatite columns and the determination of radioactivity in the fractions containing single-stranded DNA and dsDNA was performed ${ }^{47}(n=3)$. The percent of maturation of replicons was calculated by the following equation: (radioactivity in dsDNA/total radioactivity) $\times 100 \%$. The data were obtained with single $\operatorname{Rev} 3^{+I+}$ and $\operatorname{Rev} 3^{-1-}$ MEF lines and two Rev3 ${ }^{+1-}$ MEF lines.

Western blotting. In each well of a six-well plate, $2.5 \times 10^{5}$ exponentially growing cells were seeded in MEF medium and cultured overnight. Then, the cells were mock treated with the solvent THF or exposed to $500 \mathrm{nmol}$ BPDE for $15 \mathrm{~min}$ in serum-free medium at $37^{\circ} \mathrm{C}$. After rinsing the cells with PBS, cells were cultured in MEF medium. At different times after treatment, up to $12 \mathrm{~h}$, cells were lysed using Laemmli buffer. The proteins were separated by SDS-polyacrylamide gel electrophoresis and blotted onto nitrocellulose membranes (Hybond-C extra; Amersham Biosciences). The membranes were incubated overnight at $4{ }^{\circ} \mathrm{C}$ with antibodies specific for $\gamma \mathrm{H} 2 \mathrm{AX}$ (S129) (mouse monoclonal; Millipore; cat. no. 05-0636; dilution 1:1,000), phosphorylated Chk1 (at serine 345; rabbit polyclonal; Cell Signaling; cat. no. 2348; dilution 1:1,000), phosphorylated Rpa32 (at serines 4/8; rabbit polyclonal; Bethyl; cat. no. A300-245A; dilution 1:1,000), total Rpa (rat polyclonal; Cell Signaling; cat. no. 2208; dilution 1:1,000) and $\beta$-actin (mouse monoclonal; Oncogene; cat. no. CP01; dilution 1:20,000). After incubation with appropriate secondary antibodies conjugated to peroxidase (Bio-Rad), proteins were visualized using enhanced chemoluminescence detection. Uncropped scans with indicated size markers are shown in Supplementary Fig. 5.

Statistical analysis. The probability of finding two mutations in a cohort of 103 in each gene was calculated by the Poisson test. We took into account the presence of two alleles and estimated gene mutation rates based on recent exome sequencing data $^{54}$. Moreover, a conservative Bonferroni correction was done for multiple testing.

Statistical significance of differences in motoneuron counts between the wt mice and the mutant mice were determined by the unpaired $t$-test. The significance of differences of the hindbrain volume and subarachnoid space between the Rev $3 l$ heterozygous and the wt mice was calculated by the comparative $t$-test. Significance in sensitivities of MEF lines to BPDE and in replication fork progression after exposure to BPDE was determined using the unpaired $t$-test, $\sigma=0.90$. A $P$ value smaller than 0.05 was considered to be statistically significant $\left({ }^{*}\right)$, and $P$ values smaller than $\left.0.01{ }^{* *}\right)$ or $0.001{ }^{(* *)}$ were considered highly significant.

\section{References}

1. von Graefe, A. In: Handbuch der Gesammten Augenheilkunde. (eds von Graefe, A. \& Saemisch, T.) Vol. 6, 148 (W Engelmann, 1880).

2. Möbius, P. J. Ueber angeborene doppelseitige Abducens-Facialis-Lähmung. Münchener Medicinische Wochenschrift. About Congenital 35, 91-94 (1888).

3. Verzijl, H. T., van der Zwaag, B., Lammens, M., ten Donkelaar, H. J. \& Padberg, G. W. The neuropathology of hereditary congenital facial palsy vs Möbius syndrome. Neurology 64, 649-653 (2005).

4. Assaf, A. A. Congenital innervation dysgenesis syndrome (CID)/congenital cranial dysinnervation disorders (CCDDs). Eye (Lond) 25, 1251-1261 (2011).

5. Rucker, J. C. et al. Characterization of ocular motor deficits in congenital facial weakness: Moebius and related syndromes. Brain 137, 1068-1079 (2014).

6. MacKinnon, S. et al. Diagnostic distinctions and genetic analysis of patients diagnosed with moebius syndrome. Ophthalmol 121, 1461-1468 (2014).
7. Ventura, B. V. et al. Profile of ocular and systemic characteristics in Möbius sequence patients from Brazil and Italy. Arq. Bras. Oftalmol. 75, 202-206 (2012).

8. Puvabanditsin, S., Garrow, E., Augustin, G., Titapiwatanakul, R. \& Kuniyoshi, K. M. Poland-Möbius syndrome and cocaine abuse: a relook at vascular etiology. Pediatr. Neurol. 32, 285-287 (2005).

9. Weaver, D. D. Subclavian artery supply disruption sequence: hypothesis of a vascular etiology for Poland, Klippel-Feil, and Möbius anomalies. Am. J. Med. Genet. 23, 903-918 (1986)

10. Legum, C., Godel, V. \& Nemet, P. Heterogeneity and pleiotropism in the Moebius syndrome. Clin. Genet. 20, 254-259 (1981).

11. Tischfield, M. A. et al. HOXA1 mutations disrupt human brainstem, inner ear, cardiovascular and cognitive development. Nat. Genet. 37, 1035-1037 (2005).

12. Ziter, F. A., Wiser, W. C. \& Robinson, A. Three-generation pedigree of a Möbius syndrome variant with chromosome translocation. Arch. Neurol. 34, 437-442 (1977).

13. Schröder, J. C. et al. A boy with homozygous microdeletion of NEUROG1 presents with a congenital cranial dysinnervation disorder [Moebius syndrome variant]. Behav. Brain Funct. 9, 7 (2013).

14. Webb, B. D. et al. HOXB1 founder mutation in humans recapitulates the phenotype of Hoxb1 ${ }^{-1-}$ mice. Am. J. Hum. Genet. 91, 171-179 (2012).

15. Veltman, J. A. \& Brunner, H. G. De novo mutations in human genetic disease. Nat. Rev. Genet. 13, 565-575 (2012).

16. Pedraza, S. et al. MRI findings in Möbius syndrome: correlation with clinical features. Neurology 55, 1058-1060 (2000).

17. Verzijl, H. T., van der Zwaag, B., Cruysberg, J. R. \& Padberg, G. W. Möbius syndrome redefined: a syndrome of rhombencephalic maldevelopment. Neurology 61, 327-333 (2003).

18. Kremer, H. et al. Localization of a gene for Möbius syndrome to chromosome 3q by linkage analysis in a Dutch family. Hum. Mol. Genet. 5, 1367-1371 (1996).

19. Verzijl, H. T. et al. A second gene for autosomal dominant Möbius syndrome is localized to chromosome 10q, in a Dutch family. Am. J. Hum. Genet. 65, 752-756 (1999).

20. van der Zwaag, B. et al. PLEXIN-D1, a novel plexin family member, is expressed in vascular endothelium and the central nervous system during mouse embryogenesis. Dev. Dyn. 225, 336-343 (2002).

21. van der Zwaag, B. et al. Sequence analysis of the PLEXIN-D1 gene in Möbius syndrome patients. Pediatr. Neurol. 31, 114-118 (2004).

22. Romanieko, P. J. \& Camerini-Otero, R. D. The mouse Spol1 gene is required for meiotic chromosome synapsis. Mol. Cell 6, 975-987 (2000).

23. Halbritter, J. et al. Defects in the IFT-B component IFT172 cause Jeune and Mainzer-Saldino syndromes in humans. Am. J. Hum. Genet. 5, 915-925 (2013).

24. Hota, P. K. \& Buck, M. Plexin structures are coming: opportunities for multilevel investigations of semaphorin guidance receptors, their cell signaling mechanisms, and functions. Cell. Mol. Life Sci. 22, 3765-3805 (2012).

25. Petrovski, S., Wang, Q., Heinzen, E. L., Allen, A. S. \& Goldstein, D. B. Genic intolerance to functional variation and the interpretation of personal genomes. PLoS Genet. 9, e1003709 (2013).

26. Tamagnone, L. et al. Plexins are a large family of receptors for transmembrane, secreted, and GPI-anchored semaphorins in vertebrates. Cell 99, 71-80 (1999).

27. Torres-Vázquez, J. et al. Semaphorin-plexin signaling guides patterning of the developing vasculature. Dev. Cell 7, 117-123 (2004).

28. Holl, E. K. et al. Plexin-B2 and plexin-D1 in dendritic cells: expression and IL-12/IL-23p40 production. PLoS ONE 7, e43333 (2012).

29. Carmeliet, P. \& Tessier-Lavigne, M. Common mechanisms of nerve and blood vessel wiring. Nature 436, 193-200 (2005).

30. Chauvet, S. et al. Gating of Sema3E/PlexinD1 signaling by neuropilin-1 switches axonal repulsion to attraction during brain development. Neuron 56, 807-822 (2007).

31. Ding, J. B., Oh, W. J., Sabatini, B. L. \& Gu, C. Semaphorin 3E-Plexin-D1 signaling controls pathway-specific synapse formation in the striatum. Nat. Neurosci. 15, 215-223 (2011).

32. Gitler, A. D., Lu, M. M. \& Epstein, J. A. PlexinD1 and semaphorin signaling are required in endothelial cells for cardiovascular development. Dev. Cell 7, 107-116 (2004).

33. Auclair, F., Valdés, N. \& Marchand, R. Rhombomere-specific origin of branchial and visceral motoneurons of the facial nerve in the rat embryo. J. Comp. Neurol. 369, 451-461 (1996).

34. Lumsden, A. \& Keynes, R. Segmental patterns of neuronal development in the chick hindbrain. Nature 337, 424-428 (1989).

35. Studer, M., Lumsden, A., Ariza-McNaughton, L., Bradley, A. \& Krumlauf, R Altered segmental identity and abnormal migration of motor neurons in mice lacking Hoxb-1. Nature 384, 630-634 (1996).

36. Van Sloun, P. P. et al. Involvement of mouse Rev3 in tolerance of endogenous and exogenous DNA damage. Mol. Cell. Biol. 22, 2159-2169 (2002). 
37. Lin, W., Wu, X. \& Wang, Z. A full-length cDNA of hREV3 is predicted to encode DNA polymerase zeta for damage-induced mutagenesis in humans. Mutat. Res. 433, 89-98 (1999).

38. Lange, S. S., Wittschieben, J. P. \& Wood, R. D. DNA polymerase zeta is required for proliferation of normal mammalian cells. Nucleic Acids Res. 40, 4473-4482 (2012).

39. Sale, J. E. Competition, collaboration and coordination--determining how cells bypass DNA damage. J. Cell. Sci. 125, 1633-1643 (2012).

40. Jackson, S. P. \& Bartek, J. The DNA-damage response in human biology and disease. Nature 461, 1071-1078 (2009).

41. Zhang, Y. et al. Tie2Cre-mediated inactivation of plexinD1 results in congenital heart, vascular and skeletal defects. Dev. Biol. 325, 82-93 (2009).

42. Kanda, T. et al. PlexinD1 deficiency induces defects in axial skeletal morphogenesis. J. Cell. Biochem. 101, 1329-1337 (2007).

43. Carta, A., Mora, P., Neri, A., Favilla, S. \& Sadun, A. A. Ophthalmologic and systemic features in möbius syndrome an italian case series. Ophthalmol 118, 1518-1523 (2011).

44. Briegel, W. Neuropsychiatric findings of Möbius sequence -- a review. Clin. Genet. 70, 91-97 (2006).

45. Gilissen, C. et al. Genome sequencing identifies major causes of severe intellectual disability. Nature 511, 344-347 (2014).

46. Roscioli, T. et al. Mutations in ISPD cause Walker-Warburg syndrome and defective glycosylation of $\alpha$-dystroglycan. Nat. Genet. 44, 581-585 (2012).

47. Jansen, J. G. et al. Mammalian polymerase zeta is essential for post-replication repair of UV-induced DNA lesions. DNA Repair (Amst). 8, 1444-1451 (2009).

48. de Brouwer, A. P., van Bokhoven, H. \& Kremer, H. Comparison of 12 reference genes for normalization of gene expression levels in Epstein-Barr virustransformed lymphoblastoid cell lines and fibroblasts. Mol. Diagn. Ther. 10, 197-204 (2006).

49. Pfaffl, M. W. A new mathematical model for relative quantification in real-time RT-PCR. Nucleic Acids Res. 29, e45 (2001).

50. Theiler, K. The House Mouse: Atlas of Embryonic Development (Springer, 1989).

51. Davenport, H. A. Histological and Histochemical Techniques (W.B. Saunders, 1960).

52. Schmuck, M. et al. Automatic counting and positioning of 5-bromo-2deoxyuridine (BrdU) positive cells in cortical layers of rat brain slices. Neurotox 10, 1657-1664 (2014).

53. Bock, D. D. et al. Work anatomy and in vivo physiology of visual cortical neurons. Nature 471, 177-182 (2011).

54. Samocha, K. E., Robinson, E. B. \& Sanders, S. J. A framework for the interpretation of de novo mutation in human disease. Nat. Genet. 9, 944-9502014.

\section{Acknowledgements}

We thank the patients and their parents for participating in this study. We thank Luis Puelles for financial support. We also thank Maria Carmen Morga, Silvia Albert, Yike
Huang and Daniel Lopo Polla for the technical assistance and Antonia Alonso for the morphological analysis assessment. L.T.-R. is a recipient of grants from the Fundación Séneca fellowship (04548/germ/06), EMBO short-term fellowship 200-2011, the IBRO Project InEurope grants programme and the Fundación Cultural Privada EstebanRomero. Research in the group of H.v.B. is supported by the EU FP7 Large-Scale Integrating Project Genetic and Epigenetic Networks in Cognitive Dysfunction (241995). H.K. is supported by the Scientific and Technological Research Council of Turkey (TUBITAK), grants 108S418 and 112S398 and CRANIRARE consortia of the European Research Area Network (E-RARE). The work of A.C. was supported by a grant from the Italian Association of Möbius Syndrome (AISMO, www.moebius-italia.it). A.T.-S. was funded in part by the Dutch Cancer Society and A.H. by the Netherlands Organization for Health Research and Development (ZonMW 916-12-095).

\section{Author contributions}

H.v.B., G.W.P., A.C. and N.d.W. conceived the project and planned the experiments H.v.B., T.R., A.H., U.A., A.P.M.d.B. and L.T.-R. performed exome sequencing. L.T.-R., E.v.B., Z.I. and L.S. performed the validation experiments. A.T.-S. and J.G.J. performed mouse breeding and DNA damage experiments. M.K.S. and J.A.E. performed mouse breeding and conducted brain dissections. H.K., H.V., C.E., H.B., A.P.A. and G.W.P. clinically characterized the Möbius syndrome cases and collected blood samples and contributed to drafting the manuscript. A.H. and C.G. performed exome sequencing statistical analysis. L.T.-R. performed morphologic brain experiments. L.T.-R., F.M. and P.A. analysed and interpreted the data.

\section{Additional information}

Accession numbers: The novel exome sequencing data generated in this study has been deposited at the European Genome-phenome Archive (http://www.ebi.ac.uk/ega/) under accession numbers EGAS00001001250.

Supplementary Information accompanies this paper at http://www.nature.com/ naturecommunications

Competing financial interests: The authors declare no competing financial interests

Reprints and permission information is available online at http://npg.nature.com/ reprintsandpermissions/

How to cite this article: Tomas-Roca, L. et al. De novo mutations in PLXND1 and REV3L cause Möbius syndrome. Nat. Commun. 6:7199 doi: 10.1038/ncomms8199 (2015).

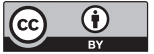

This work is licensed under a Creative Commons Attribution 4.0 International License. The images or other third party material in this article are included in the article's Creative Commons license, unless indicated otherwise in the credit line; if the material is not included under the Creative Commons license, users will need to obtain permission from the license holder to reproduce the material. To view a copy of this license, visit http://creativecommons.org/licenses/by/4.0/ 